\title{
Atomic force microscopy and laser confocal scanning microscopy analysis of callose fibers developed from protoplasts of embryogenic cells of a conifer
}

Takeshi Fukumoto $^{1}$, Noriko Hayashi ${ }^{2}$, Hamako Sasamoto ${ }^{1}$

${ }^{1}$ Faculty of Environment and Information Sciences, Yokohama National University, Yokohama 240-8501, Kanagawa, Japan

${ }^{2}$ Forestry and Forest Products Research Institute, Tsukuba 305-8687, Ibaraki, Japan

To whom correspondence should be addressed:

E-mail: sasamoto@ynu.ac.jp

Tel: +81 (45) 3394414

Fax: +81 (45) 3394414

Abstract. Efficiency of novel fiber formation was much improved in protoplast culture of embryogenic cells (ECs) of a conifer, Larix leptolepis (Sieb. et Zucc.) Gord., by pre-culturing ECs in a medium containing a high concentration of glutamine (13.7 mM). The fibrillar substructures of large and elongated fibers of protoplasts isolated from Larix ECs were investigated by laser confocal scanning microscopy (LCSM) after Aniline Blue staining and atomic force microscopy (AFM) using a micromanipulator without any pre-treatment. Fibers were composed of bundles of fibrils and subfibrils, whose diameters were defined as $0.7 \mu \mathrm{m}$ and $0.17 \mu \mathrm{m}$, respectively, by image analysis after LCSM and AFM. These fibers were proven to be composed of callose by using specific degrading enzymes for $\beta$-1,4-glucan and $\beta$-1,3-glucan. 
Key words: AFM - $\beta$-1,3-Glucan - Embryogenic cells - Larix - LCSM - Protoplast fiber

\section{Introduction}

Previously, we found novel callose fiber formation in protoplast cultures of Betula platyphylla and Larix leptolepis in the media containing high concentrations of calcium and magnesium ions, respectively (Sasamoto et al. 2003). Aniline Blue staining has mainly been used for detection of callose as a component of large and elongated fibers developed in protoplast cultures of both a broad leaved tree, B. platyphylla, and a conifer, L. leptolepis. In the latter, protoplast-fibers were detected much earlier than in the former, and helical fibril structure in a big fiber of $10 \mu \mathrm{m}$ width was shown by transmission electron microscopy. Recently, atomic force microscopy (AFM) was introduced for exploring the microfibrillar structures of cellulose fibers on the cell wall surface in situ (Pesacreta et al. 1997). Cellulose is a fiber forming glucan with $\beta$-1,4-linkage. To elucidate the regulatory mechanism for forming callose fiber with $\beta$-1,3-linkage, we need to study further the substructures of the protoplast fibers. In this study, we performed laser confocal scanning microscopy (LCSM) after staining with Aniline Blue, and AFM to clarify the substructures of novel elongated protoplast fibers of embryogenic cells of L. leptolepis, after much improvement of protoplast isolation 
efficiency. The callose component was further confirmed by using specific glucanases. The characteristics of the callose fibers, and cellulose fibers deposited on the cell surface of herbaceous plants are also discussed.

\section{Materials and methods}

Suspension culture of embryogenic cells (ECs)

The ECs of Larix leptolepis were obtained and subcultured every 3 weeks as described previously (Ogita et al. 1997; Sasamoto et al. 2003). Briefly, ECs were cultured in modified $\mathrm{CD}(\mathrm{mCD})$ medium, containing a half concentration of ammonium nitrate (5 $\mathrm{mM}), 7 \mu \mathrm{M}$ 2,4-dichlorophenoxyacetic acid (2,4-D), $3 \mu \mathrm{M}$ 6-benzyladenine (BA), 0.09 $\mathrm{M}$ sucrose, and $4.1 \mathrm{mM}$ of L-glutamine. The glutamine concentration in the medium was increased up to $13.7 \mathrm{mM}$ when cited in the text. The medium $\mathrm{pH}$ was adjusted at 5.8 before autoclaving at $121^{\circ} \mathrm{C}$ for $20 \mathrm{~min}$. These ECs were maintained on the rotary shaker at $100 \mathrm{rpm}$ speed at $25^{\circ} \mathrm{C}$ under the $16 \mathrm{~h}$ of light photoperiod $\left(120 \mu \mathrm{mol} \mathrm{m} \mathrm{m}^{-2}\right)$ and used for the protoplast isolation. Cells were observed under an inverted microscope (Olympus; CK40).

Protoplast isolation and culture for fiber formation 
Prior to the protoplast isolation, the ECs were collected on a $42 \mu \mathrm{m}$ nylon mesh and washed with 0.4 M mannitol solution. Protoplasts from ECs, cultured in $4.1 \mathrm{mM}$ glutamine containing mCD medium, were isolated by incubation with $1 \%$ Cellulase RS (Yakult Honsya Co.,Ltd.) and 0.25\% Pectolyase Y-23 (Kyowa Chemical Products Co.,Ltd.) in $0.4 \mathrm{M}$ mannitol for 6 to $8 \mathrm{~h}$ on a rotary shaker at $80 \mathrm{rpm}$ speed. On the other hand, protoplasts from ECs, cultured in $13.7 \mathrm{mM}$ glutamine containing $\mathrm{mCD}$ medium, were isolated by incubation with $1 \%$ Cellulase RS and $0.05 \%$ Pectolyase Y-23 in $0.4 \mathrm{M}$ mannitol for 60 to $90 \mathrm{~min}$ on a rotary shaker at $80 \mathrm{rpm}$ speed. In the preliminary experiments, 0.1 and $0.25 \%$ of Pectolyase Y-23 were also tested. After passing through a $42 \mu \mathrm{m}$ nylon mesh, protoplasts were washed 3 times with $0.4 \mathrm{M}$ mannitol solution by centrifugation at $100 \times \mathrm{g}$ for $4 \mathrm{~min}$. Culture was conducted as described by Sasamoto et al. (2003). Namely, the ammonium nitrate-free MS (Murashige and Skoog 1962) medium containing $10 \mu \mathrm{M}$ each of 2,4-D and BA, 0.09 to $0.27 \mathrm{M}$ sucrose, and $50 \mathrm{mM}$ $\mathrm{MgCl}_{2}$ at $\mathrm{pH} 5.8$ was used for induction of fiber-structures from protoplasts. Final cell density was adjusted to 2.5 to $5 \times 10^{4}$ protoplasts $\mathrm{ml}^{-1}$. Fifty micro-liters of medium in a 96-well culture plate (Falcon no. 3075), $400 \mu \mathrm{l}$ of medium in a 24 -well culture plate (Falcon no. 3047), or $1000 \mu \mathrm{l}$ of medium in a 6-well culture plate (Iwaki code. 3810-006) were used. Sterilized water was distributed into the space between wells to maintain humidity, and the plate was sealed with Parafilm ${ }^{\circledR}$. All of these procedures were performed under sterile conditions. Protoplasts were cultured in the dark at $28^{\circ} \mathrm{C}$ using a $\mathrm{CO}_{2}$ incubator without supply of $\mathrm{CO}_{2}$. The protoplasts were observed under an inverted microscope periodically. 


\section{Purification of fibers by micromanipulation technique}

Protoplast fibers were purified using a micromanipulator (Narishige MM-188 or MO-202 with a microinjector IM-188) attached to an inverted microscope (Olympus; CK40). Ten-microliters of precision disposable micropipettes (Drummond Scientific Co., Broomall, PA; Cat. No. 2-000-010) were converted by the method described as Ogita et al. (1999). Fiber-structures were picked up and washed by transferring several times to $500 \mu \mathrm{l}$ of fresh sterile deionized water in four-well plastic culture plates (Falcon no. 3654) in order to remove the culture medium. Purified fibers in water were used as fiber solution for LCSM and AFM observation, and degradation with specific glucanases.

Fluorescent staining of fibers with Aniline Blue and observation using laser confocal scanning microscopy (LCSM)

Fiber-structures originating from protoplasts at about 2 weeks of culture, were stained with Aniline Blue. Five micro-liters of Aniline Blue solution (0.05\%) in phosphate buffer ( $\mathrm{pH} 8.5$ ) was dispensed into $55 \mu \mathrm{l}$ of culture medium with fiber-structures in a 96-well culture plate. They were observed under an inverted fluorescent microscope (Olympus IX-71, U excitation with filter BP 330-385 and with absorption filter BA 420). On the other hand, $50 \mu \mathrm{l}$ of fiber solution, purified by the micromanipulation 
technique, was stained by same method described above and observed by laser confocal scanning microscopy (Bio-rad, Radiance 2100, excitation wavelength of blue laser of $405 \mathrm{~nm})$. Obtained images were used for the image analysis.

Degradation of fiber-structures by enzymatic treatment

Each of three glucosidases with different specificity was dissolved in deionized water and used as an enzymatic solution as follows; $\beta$-1,4-glucan cellobiohydrolase I (CBH I) [EC 3.2.1.91], crude cellulase from Trichoderma viride (Hayashi et al. 1998a, b), laminarinase [EC 3.2.1.6] (Sigma Chemical Co. Ltd. St. Louis, MO). Twenty five micro-liters each of fiber solution and an enzymatic solution were mixed as a reaction mixture and incubated at $28^{\circ} \mathrm{C}$. Bacterial (Acetobacter xylinum) cellulose and cotton cellulose (Hayashi et al. 1998b) were used as a control. The amount of protein in these enzymatic solutions was quantified (Bradford 1976), with bovine serum albumin (BSA) as a standard. Fibers were observed for degradation under an inverted microscope. Fiber degradation was confirmed after staining with Aniline Blue solution. Experiments were repeated once.

Atomic force microscopy (AFM) observation

The fiber-structures were observed under an atomic force microscope (Nanoscope III; Digital Instruments) equipped with a scanner with a $10 \mu \mathrm{m}$ range (E-scanner). Purified 
fiber solution was picked up with a micromanipulator and placed onto cleaned, round, glass coverslips $10 \mathrm{~mm}$ in diameter using a micromanipulator and glued onto metal discs of $10 \mathrm{~mm}$ diameter. After drying of the surface water at room temperature, the metal disc was placed on the stage of AFM. All images $(400 \times 400$ pixels $)$ were obtained using a tapping mode in air. The cantilever used was $130 \mu \mathrm{m}$ in length and had an unmodified silicon nitride tip with a spring constant of $40 \mathrm{Nm}^{-1}$. The scan rate was $1.5 \mathrm{~Hz}$ and the scan angle was varied to obtain the optimum contrast. Obtained images were used for the image analysis.

Image analysis

To predict the substructures of these fibers, we analyzed the LCSM and AFM images by using the image analysis program, Image J (NIH, USA). Plot profile data was obtained from the prediction area indicated in figures in the Results. Peak distances were calculated by use of Image $\mathrm{J}$, and averaged as a diameter of these fibers or (sub)fibrils.

\section{Results}

Effect of glutamine on embryogenic cell morphology and improvement of protoplast isolation 
Protoplasts from the embyogenic cells cultured in mCD liquid medium containing 4.1 mM glutamine were isolated by a method described previously (Sasamoto et al. 2003), which took about 6 to $8 \mathrm{~h}$ for protoplast isolation with $0.25 \%$ of Pectolyase Y-23 and some parts remained undigested. In ECs cultured in the presence of $13.7 \mathrm{mM}$ glutamine, the size of embryogenic cell masses became smaller than that cultured in the presence of 4.1 $\mathrm{mM}$ glutamine. In addition, protoplasts were isolated more effectively and rapidly by using ECs, cultured at $13.7 \mathrm{mM}$ glutamine. Both time (1/6) of incubation and concentration (1/5) of Pectolyase Y-23 for protoplast isolation were reduced by culturing the ECs in the presence of $13.7 \mathrm{mM}$ glutamine.

Observation of fibers from Larix protoplast

Immediately after protoplast isolation, isolated protoplasts were not stainable with Aniline Blue. After 1 to 2 weeks of culture in the medium containing $50 \mathrm{mM}$ of $\mathrm{MgCl}_{2}$, helical fiber-structures were developed from protoplasts (Fig. 1). These structures were obtained repeatedly in protoplast culture of both ECs cultured at $4.1 \mathrm{mM}$ and $13.7 \mathrm{mM}$ of glutamine. Fiber formation was also observed at all sucrose concentrations examined. About $0.4 \%$ of protoplasts formed fibers after 14 days of culture at $0.18 \mathrm{M}$ of sucrose in the medium. As shown in Fig. 1a and 1b, a fiber-structure was developed from a single part of big spherical protoplasts. The fiber portion was stainable with Aniline Blue, but the cell surface was not. Also, these fiber-structures were often highly coiled and elongated about a couple of millimeters in length, and fluoresced after Aniline Blue 
staining (Fig. 1c and 1d).

Enzymatic degradation analysis

$\mathrm{CBH} 1$, crude cellulase and laminarinase treatment for $24 \mathrm{~h}$ at $28^{\circ} \mathrm{C}$ showed a distinctive degradation pattern between Larix protoplast fiber, and cotton and bacterial cellulose fibers (Fig. 2). CBH I did not digest protoplast fibers from L. leptolepis (Fig. 2a), while cotton cellulose (Fig. 2d) and bacterial cellulose (Fig. 2g) were digested. By contrast, Larix fibers were completely cleaved with laminarinase (Fig. 1c), but cotton (Fig. 2f) and bacterial cellulose (Fig. 2i) remained undigested. Crude cellulase digested all three substrates. After $48 \mathrm{~h}$ treatment (data not shown), Larix fibers were slightly cleaved to make short fibers by CBH I solution. Laminarinase partially digested the cotton and bacterial cellulose after $48 \mathrm{~h}$, while $\mathrm{CBH}$ I and crude cellulase completely digested these substrates within $48 \mathrm{~h}$. The Bradford protein assay indicated that 50 micro-liters of each enzymatic solution contained about $50 \mu \mathrm{g}$ of $\mathrm{CBH}$ I, $25 \mu \mathrm{g}$ of crude cellulase, and 12.5 $\mu \mathrm{g}$ laminarinase, respectively.

\section{Analysis of LCSM and AFM images}

After purification of fibers by using a micro-manipulator, the substructures of these fibers were observed by LCSM and AFM. LSCM image shows that the fiber-structures, developed from protoplasts isolated from ECs of L. leptolepis, were composed of 
bundle of fibrils (Fig. 3a). Peak distances were calculated and averaged to determine the diameter of these fibers (Fig 3b). Furthermore, plot profile data was obtained from the prediction area indicated by ramdomly selected red line in Fig. 3a. After the image analysis on LCSM image, the fibrils had a diameter calculated to be $0.63 \pm 0.24$ (S.D.) (Fig. 3b). AFM revealed a definitive bundle of fibrils. Plot profile data was obtained from the prediction area indicated by the white line in Fig. 4a. Also, AFM image analysis showed that the fibril with a diameter of $0.70 \pm 0.1 \mu \mathrm{m}$ (S.D.), consisted of a bundle of several subfibrils with a diameter of $0.17 \pm 0.07 \mu \mathrm{m}$ (S.D) (Fig. 4b).

\section{Discussion}

The efficiency of novel fiber formation was much improved by refining the condition of protoplast isolation from Larix ECs by culturing ECs in high glutamine containing medium. Viable protoplasts could be isolated using a low concentration of Pectolyase Y-23 and brief incubation with enzymes. The efficiency of fiber formation from Larix protoplasts, isolated from ECs cultured in high glutamine, was $0.4 \%$ of plated protoplasts, which was similar to the value obtained in a previous study in which ECs were cultured in the presence of $4.1 \mathrm{mM}$ glutamine, in the same medium composition, containing $50 \mathrm{mM}$ of $\mathrm{MgCl}_{2}$, and at the same protoplast density. Such a high concentration of glutamine, $13.7 \mathrm{mM}$, is also essential for maintenance of embryogenic cell culture of another Japanese conifer, Cryptomeria japonica (Ogita et al. 2001) and 
the ECs in liquid culture (Sasamoto et al. 2002) were small like the ECs of Larix. Protoplast fibers were also observed in the Japanese cedar (Sasamoto et al. 2001). As these fibers can be first observed within one week of culture, the ECs of these conifers might be a good source for mass production of protoplast fibers and for clarifying the process of fiber formation. By contrast, leaf protoplasts of Betula platyphylla form elongated fibers after more than two months of culture in the medium containing 100 $\mathrm{mM}$ of $\mathrm{CaCl}_{2}$ (Sasamoto et al. 2003).

Elongated and highly coiled fiber structures, which originated from a special part of cell surface, were stained with Aniline Blue. However, the cell surface was not stained with this fluorescent dye. Hahne et al. (1983) and Hoffman (1996) reported that the short ribbon-like structure and part of the cell surface fluoresced with Calcofluor White in tobacco protoplast culture, and described it as cellulose by enzymatic treatment. However, they are different from the fibers found in the protoplast cultures of Larix ECs, which are very elongated and proven to be a callose fiber. In our study, we mainly used Aniline Blue staining specific for callose, and used enzymatic treatment to further confirm the callose, $\beta$-1,3-glucan component of the elongated protoplast fiber. Laminarinase digested the Larix fiber while CBH I did not. CBH I, a specific exo-1,4- $\beta$-glucanase, catalyses the hydrolysis of cellulose into cellobiose by attacking crystallized $\beta$-1,4-linked polysaccharide (cellulose). Although crude cellulase and laminarinase have both activities of $\beta$-1,4- and $\beta$-1,3-glucanase, laminarinase has more specific activity for $\beta$-1,3-glucan degradation. On the other hand, in tobacco BY-2 protoplast culture, we could obtain elongated fibers in the medium at a high $\mathrm{Mg}^{2+}$ ion 
concentration but the efficiency was still very low (Fukumoto et al. 2004). The fiber-structures formed in the protoplast culture of L. leptolepis, were composed of $\beta$-1,3-glucan (callose).

Sasamoto et al. (2003) observed the helical bundle of fibrils, in Larix, consisting of fibers $10 \mu \mathrm{m}$ in diameter using a transmission electron microscope. In the present study, the presence of fibril structures was supported by LCSM image analysis and by the AFM, and their size was determined to be $0.7 \mu \mathrm{m}$ in diameter. Furthermore, subfibril structure was revealed by analysis of AFM image. Although, there have been no reports of large and elongated fiber structures composed of callose except for the protoplast fibers, they can be compared to the cellulose fibers, which usually deposit on the surface of cell walls (Hayashi et al. 1998a, b). By AFM analysis of the cotton fiber cell wall surfaces (Pesacreta et al. 1997) using the same tapping mode in air as in the present study, the size of the cellulose fiber without any reagent treatment (A-fiber) was similar to that of subfibrils of protoplast fibers. Among the three methods of observation of the protoplast fibers, AFM with micromanipulation technique might be the most practical method for observation of fibrillar substructures without staining with fluorescent dye. Further studies are underway on fine structures of subfibrils of protoplast fiber and on its extruding part of cell surface, by TEM and AFM observation.

\section{Acknowledgements}

Thanks are due to Drs. A. Takebe and N. Oba for their kind help with image analysis using LCSM. T.F. is a research fellow of the Japan Society for the Promotion of Science 
(JSPS) for Young Scientists.

\section{References}

Bradford MM (1976) A rapid and sensitive method for the quantitation of microgram quantities of protein utilizing the principle of protein-dye binding. Anal. Biochem. $72: 248-254$

Cheadle VI, Gifford EM, Esau K (1953) A staining technique for phloem and contiguous tissue. Stain Technol. 28:49-53

Fukumoto T, Sasamoto H, Hayashi N, Ogita S, Wakita Y, Yokota S, Yoshizawa N (2004) Development of novel large elongated fiber-structure in plant protoplast cultures. In Vitro Cell. Dev. Biol. 40:73A, 2004

Hahne G, Herth W, Hoffmann F (1983) Wall formation and cell division in Fluorescence-labelled plant protoplasts. Protoplasma 115:217-221

Hayashi N, Sugiyama J, Okano T, Ishihara M, Shimizu K (1998a) Selective degradation of cellulose Ia component in Cladophora cellulose with Trichoderma viride cellulose. Carbohydrate Res. 305:109-116

Hayashi N, Sugiyama J, Okano T, Ishihara M, Shimizu K (1998b) The enzymatic susceptibility of cellulose microfibrils of the algal-bacterial type and the cotton-ramie type. Carbohydrate Res. 305:261-269

Hoffmann F (1996) Laser microbeams for the manipulation of plant cells and subcellular structures. Plant Science 113:1-11 
Murashige T and Skoog F (1962) A revised medium for rapid growth and bioassays with tobacco tissue cultures. Physiol. Plant. 15:473-497

Ogita S, Kubo T, Fushitani M (1997) Anatomical characteristics in early embryogenesis from immature embryo of Larix leptolepis. For Res. Environ. 35:45-51

Ogita S, Sasamoto H, Kubo T (1999) Selection and microculture of single embryogenic cell clusters in Japanese conifers: Picea jezoensis, Larix leptolepis and Cryptomeria japonica. In Vitro Cell. Dev. Biol.-Plant 35:428-431

Ogita S, Sasamoto H, Yeung EC, Thope TA (2001) The effects of glutamine on the maintenance of embryogenic cultures of Cryptomeria japonica. In Vitro Cell. Dev. Biol.-Plant 37:268-273

Pesacreta TC, Carlson LC, Triplett BA (1997) Atomic force microscopy of cotton fiber cell wall surfaces in air and water: quantitative and qualitative aspects. Planta $202: 435-442$

Sasamoto H, Ogita S, Yeung, EC, Thorpe TA (2001) Protoplast culture of Larix kaempferi and Cryptomeria japonica: Regulatory factors for protoplast fiber formation and early somatic embryogenesis (in Japanese). Trans. Jpn. For. Soc. (ISSN 1341-6960) 112:660

Sasamoto H, Ogita S, Fukui M (2002) Cell engineering of Japanese conifers, Cryptomeria japonica and Larix kaempferi. In Vitro Cell. Dev. Biol. 38: p56A

Sasamoto H, Ogita S, Hayashi N, Wakita Y, Yokota S, Yoshizawa N (2003) Development of novel elongated fiber-structure in protoplast cultures of Betula platyphylla and Larix leptolepis. In Vitro Cell. Dev. Biol.-Plant 39:223-228 
Yeung EC (1984) Histological and histochemical staining procedures. In: Vasil IK (ed) Cell culture and somatic cell genetics of plants, vol. 1. Academic Press, Orlando, FL, pp 689-697

Legends of figures

Figure 1. Light and fluorescent microscopic observation of highly coiled fiber developed from single part of protoplast and large-elongated fiber-structure. Fiber structure was stained with Aniline Blue. Photographs a and c, under white light; $\mathbf{b}$ and $\mathbf{d}$, under UV fluorescent light (Bars in $\mathbf{a}$ and $\mathbf{b}=50 \mu \mathrm{m}$; Bars in $\mathbf{c}$ and $\mathbf{d}=250 \mu \mathrm{m}$ ).

Figure 2. Degradation of Larix protoplast fiber, cotton and baceterial cellulose with three kinds of $\beta$-D-glucanases. Photographs were taken at $24 \mathrm{~h}$ after enzymatic treatment of Larix protoplast fiber $(\mathbf{a}, \mathbf{b}, \mathbf{c})$, cotton cellulose $(\mathbf{d}, \mathbf{e}, \mathbf{f})$, and bacterial cellulose $(\mathbf{g}, \mathbf{h}, \mathbf{i})$. Enzymes were $\mathrm{CBH}$ I (a, d, g), crude cellulase (b, e, h) and laminarinase $(\mathbf{c}, \mathbf{f}, \mathbf{i})$.

Figure 3. LCSM image of protoplast fiber after Aniline Blue staining (a) and its image analysis (b). The bar represents $20 \mu \mathrm{m}$ length. b: Plot profile of the red line portion in a. 
$\mathrm{f} 1$ and $\mathrm{f} 2$ in magnification of a corresponds to $\mathrm{f} 1$ and $\mathrm{f} 2$ in $\mathbf{b}$, respectively.

Figure 4. AFM image of fiber-structure (a) after purification using a micromanipulator and its image analysis (b). The bar represents $5 \mu \mathrm{m}$ in $\mathbf{a} . \mathbf{b}$ : Plot profile of the white line portion in a. Letter s1 and s2 in magnification of a corresponds to s1 and s2 in $\mathbf{b}$, respectively. 